Proceedings

\title{
Comparison of the performance of two aptamers on a colorimetric assay for the quantification of fumonisin $\mathrm{B1}^{+}$
}

\author{
Vicente Antonio Mirón-Mérida *, Yadira González-Espinosa, Yun Yun Gong, Yuan Guo and \\ Francisco M Goycoolea * \\ School of Food Science and Nutrition, University of Leeds, Leeds LS2 9JT, UK; \\ * Correspondence: fsvamm@leeds.ac.uk (V.A.M.-M.); F.M.Goycoolea@leeds.ac.uk (F.M.G.) \\ + Presented at the 1st International Electronic Conference on Biosensors, 2-17 November 2020; Available \\ online: https://iecb2020.sciforum.net/.
}

Received: date; Accepted: date; Published: date

\begin{abstract}
Fumonisin B1 (FB1), a mycotoxin commonly produced by Fusarium verticillioides and classified as group 2B hazard, has been identified in various food products, hence sensitive and rapid analytical detection methods are needed. Since the first reported aptamer ( $96 \mathrm{nt}$ ss-DNA) for the highly specific molecular recognition of FB1, only 30 aptamer-based biosensors have been published. A critical point, yet commonly overlooked during the design of aptasensors, is the selection of the binding buffer. In this work, a colorimetric assay was designed by incubating a folded aptamer with FB1, and the subsequent addition of gold nanoparticles (AuNPs). The change in the aggregation profile of AuNPs by a 40-nt aptamer or a 96-nt aptamer, was tested after the addition of FB1 under different buffer conditions, where the incubation with Tris- $\mathrm{HCl}$ and $\mathrm{MgCl}_{2}$ exhibited the most favorable performances. The assay with the longest aptamer was specific to FB1 and comparable to other aptasensors with a limit of detection (LOD) of $3 \mathrm{ng} / \mathrm{mL}$ (A650/520 ratio). Additionally, the application of asymmetric flow field-flow fractionation (AF4) with multidetection allowed the analysis of the peak area $(\lambda)$ and multi-angle light scattering (MALS) with LODs of up to the $\mathrm{fg} / \mathrm{mL}$ level.
\end{abstract}

Keywords: Fumonisin B1; gold nanoparticles; aptamer; AF4

\section{Introduction}

Fumosinin refers to a family of mycotoxins from which group B is the most abundant in nature [1]. From this group, fumonisin $\mathrm{B} 1\left(\mathrm{C}_{34} \mathrm{H}_{59} \mathrm{NO}_{15}\right)$ has been monitored in multiple food commodities, due to its harmful effects on animals and classification as possible carcinogenic to humans [2]. Conventional methods for mycotoxin detection include ELISA, HPLC-FLD and LC-MS, which implicate high costs, long assays, poor portability, and experienced analysts [3]. Hence, sensitive, quick and affordable methods should be developed for the quantification of mycotoxins.

An alternative to the traditional methods is the integration of biosensing techniques with aptamers, single-stranded DNA or RNA molecules with high affinity to specific target molecules. The SELEX selection of specific aptamers for FB1 has resulted in the discovery of two main sequences namely 96-nt and 80-nt. Both sequences are already employed in about 30 aptasensors [4,5], where a critical step during their engineering is the selection of the optimum target-aptamer incubation conditions (buffer, incubation, temperature).

Gold nanoparticles (AuNP), widely utilized in multiple biosensing platforms, can be functionalized with aptamers for the colorimetric detection of FB1. This approach relies on the aggregation of AuNPs in the presence of cationic compounds [6,7]. Besides spectrophotometric 
analysis of AuNPs, asymmetric flow field flow fractionation (AF4) with multidetection (RI, MALS, DLS and UV/VIS) is a promising technique for the analysis of their interaction with aptamers during target incubation. AF4 fractionates macromolecules and particles depending on their hydrodynamic radius, and subsequently determines their distribution, based on their concentration, composition and size using the signals from the different detectors. Such separation occurs due to a parabolic laminar flow passing through a thin membrane, where AuNPs are grouped by a perpendicular flow [8]. In this work, the quantification of FB1 was assessed for two aptamers under different buffer conditions. We gained further insight on the interactions at play between the aptamer-target complex and AuNP under aggregation conditions (salt addition) using AF4 while harnessing its capacity to fractionate and characterize colloidal particles and biomacromolecules.

\section{Materials and Methods}

\subsection{Colorimetric Determination of FB1 by Functionalization of AuNP with Aptamers}

Two aptamers (40 and 96-nt) reported as specific for FB1 were individually incubated with FB1 at different concentrations (the incubation time, temperature and binding buffers will be specified for each figure), followed by the incubation with AuNP. From this mixture, $30 \mu \mathrm{L}$ were combined with $\mathrm{NaCl}$ in a 1:1 (v:v) ratio for a wavelength scan $(400-800 \mathrm{~nm})$ using a TECAN (Crailsheim, Germany) microtitre plate reader. The A520/650 ratio was determined by dividing the absorbance values at 520 and $650 \mathrm{~nm}$. The general procedure is outlined in Figure 1.

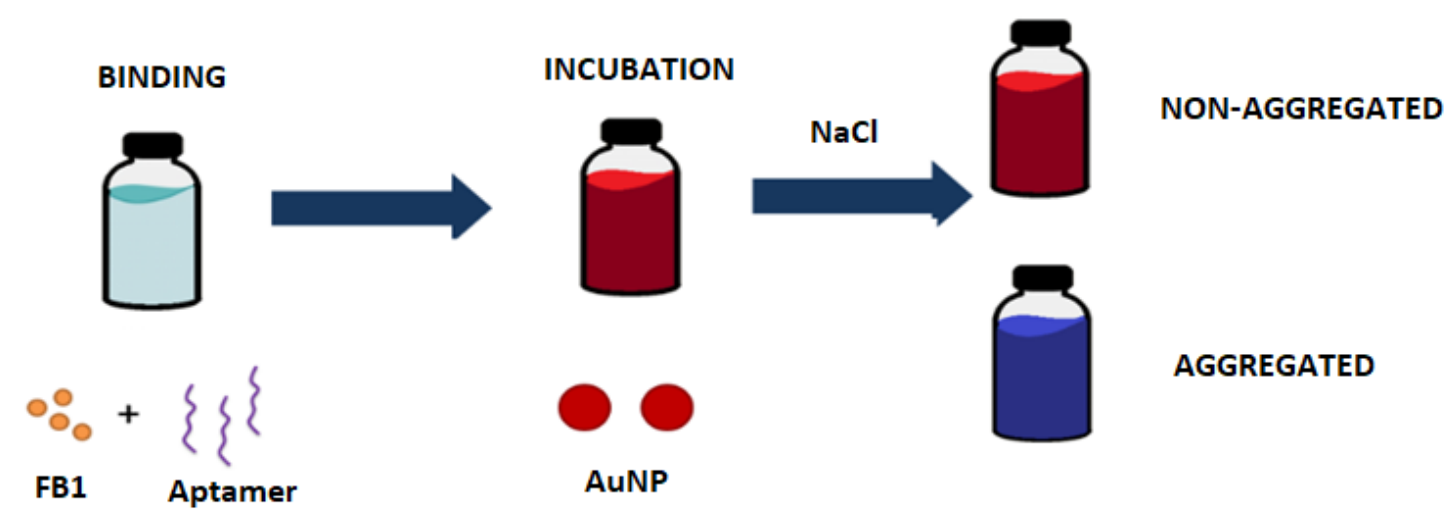

Figure 1. Colorimetric determination of FB1 with AuNP-aptamers in bulk.

\subsection{Analysis of the AuNP-FB1-Aptamer Complex by Asymmetric Flow Field Flow Fractionation (AF4)}

Asymmetric flow field-flow fractionation (AF4) measurements were performed on an AF2000 Multiflow system from Postnova Analytics $\mathrm{GmbH}$ (Landsberg am Lech, Germany). A method for the size separation of gold nanoparticles (AuNP) was developed using a regenerated cellulose membrane, with water/surfactant as carrier solvent. The optimized AF4 method consisting in an exponential decay (70 $\mathrm{min}$, cross flow: $1 \mathrm{~mL} / \mathrm{min}$, focus pump: $1.30 \mathrm{~mL} / \mathrm{min}$ ) was applied on the aggregation profile of AuNP stabilized with a FB1-aptamer complex unveiled upon addition of $\mathrm{NaCl}$ $0.2 \mathrm{M}$. The observed characteristic results at different FB1 concentrations were analyzed by UV-VIS $(600 \mathrm{~nm})$ and MALS $\left(28^{\circ}\right)$, and the peak area was calculated in Origin Pro 8.632 Bit (2012) software, considering the base line of each curve. This provide a new quantification for mycotoxins as well as an alternative method for characterizing aptamer-target interactions. 


\section{Results and Discussion}

\subsection{Quantification of FB1 with a 40-nt Aptamer}

The values of the A520/650 ratio upon incubation of a 40-nt aptamer with varying concentrations of FB1 in Tris $\mathrm{HCl} 31.1 \mathrm{mM}$ (pH 7.5), PBS buffer $12.79 \mathrm{mM}$ (pH 7.4) and a combination of both buffer media is illustrated in Figure 2. As it can be noted, an opposite and more differentiated performance was obtained when Tris $\mathrm{HCl}$ buffer was included during the binding step, which was also confirmed by the similarity of both curves containing Tris $\mathrm{HCl}$ buffer. The utilization of Tris-based buffers ( $\mathrm{pH}$ 8.2) has been tested for diluting FB1 during the incubation with this 40 -nt sequence at $37^{\circ} \mathrm{C}$, as part of the electrochemical detection with graphene-modified electrodes $[9,10]$.

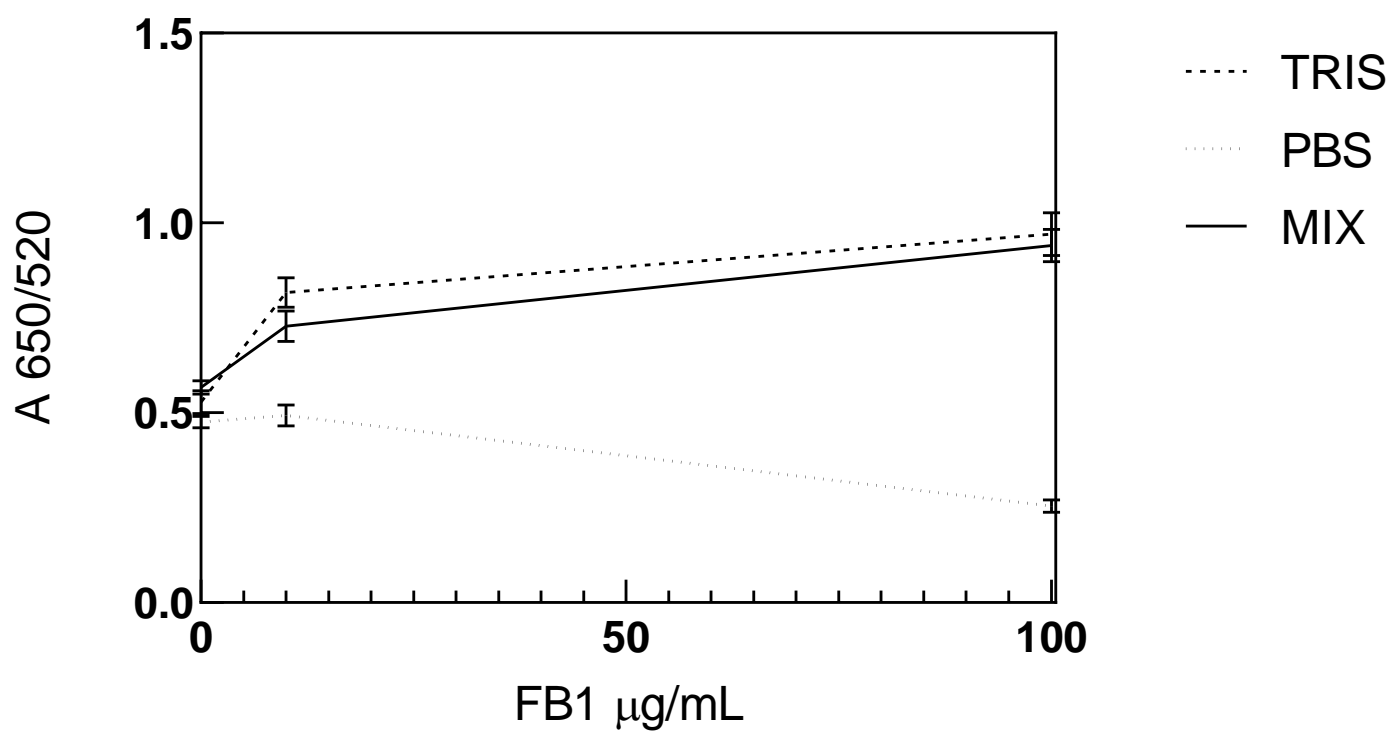

Figure 2. Effect of the incubation in Tris $\mathrm{HCl}$ (31.1 $\mathrm{mM} \mathrm{pH}$ 7.5), PBS buffer (12.79 $\mathrm{mM} \mathrm{pH} \mathrm{7.4)} \mathrm{and}$ both buffer media (MIX) on the functionalization effect of a 40-nt aptamer on AuNP at increasing FB1 concentrations upon addition of $\mathrm{NaCl} 0.4 \mathrm{M}$ (Aptamer-FB1 binding: $60 \mathrm{~min}$ at $37{ }^{\circ} \mathrm{C}$; AuNP incubation: $120 \mathrm{~min}$ at $37^{\circ} \mathrm{C}$; 500:1 Aptamer:AuNP molar ratio).

As shown in Figure 3a, the incubation of aptamers (40-nt) and FB1 in Tris $\mathrm{HCl}$ buffer resulted in a favorable sensing resolution with a limit of detection equivalent to $30 \mathrm{ng} / \mathrm{mL}$ Nevertheless, such biosensing approach was not specific for FB1 (Figure 3b), as the incubation with the same concentration of ochratoxin A (OTA) resulted in a similar A650/520 ratio to that from FB1. This result is contrary to the previously reported specificity for an electrochemical method integrating the same aptamer, whose affinity to FB1 was confirmed through specificity assays with OTA and thrombin [10]. 

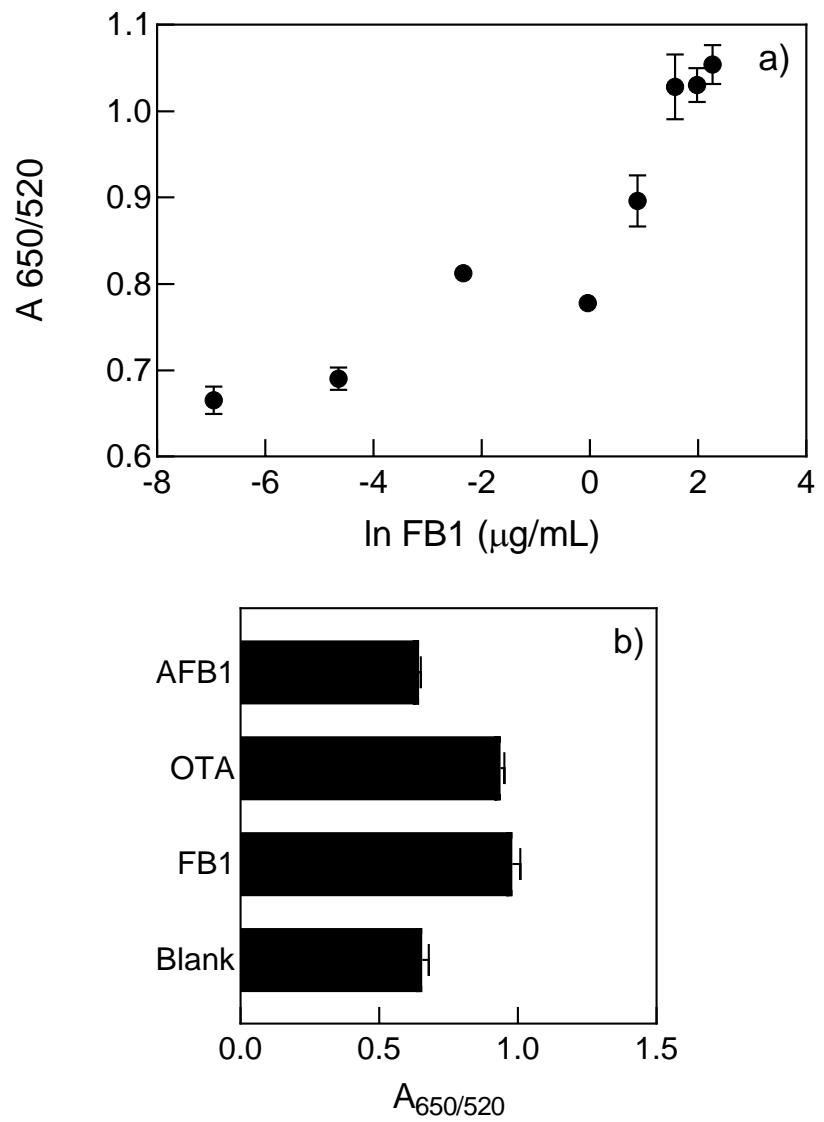

Figure 3. A650/520 ratio of AuNP functionalized with a 40-nt aptamer previously incubated with FB1 in Tris $\mathrm{HCl} 14.06 \mathrm{mM} \mathrm{pH} 7.5$ (Incubation: $60 \mathrm{~min}$ at $37{ }^{\circ} \mathrm{C}$, Functionalization: $105 \mathrm{~min}$ at $20{ }^{\circ} \mathrm{C}$, Aptamer:AuNP 500:1) after the addition of $\mathrm{NaCl} 0.4 \mathrm{M}$ (a); specificity test after the incubation with buffer (blank), FB1, aflatoxin B1 (AFB1), and ochratoxin A(OTA) at a mycotoxin concentration of 13.8 $\mu \mathrm{M}(\mathbf{b})$.

\subsection{Quantification of FB1 with a 96-nt Aptamer}

The first sequence reported for FB1 [4] was utilized in this work for the development of a colorimetric technique by completing the steps outlined in Figure 1. Despite the attempts to produce a noticeable signal at low FB1 concentrations, neither PBS nor Tris- $\mathrm{HCl}$ were successfully applied on the establishment of an aptamer-based sensing strategy. However, as displayed in Figure $4 \mathrm{a}, \mathrm{MgCl} 2$ was confirmed as an optimal buffer for a more sensitive determination of FB1, where the limit of detection based on the $\mathrm{A}_{650 / 520}$ ratio was calculated as $\sim 3 \mathrm{ng} / \mathrm{mL}$. The protective effect of the aptamerFB1 complex on the surface of AuNP was specific for the target mycotoxin, as demonstrated in Figure $4 \mathrm{~b}$, where similar aggregation profiles were found among OTA, AFB1 and the blank sample. The reduction in the aggregation of AuNP by their stabilization with an aptamer-target complex, has been explored in other molecules such as serotonin, where the binding buffer prevents the repulsion among the DNA backbone negative charges, for an improved folding and complex formation [11]. 
a)

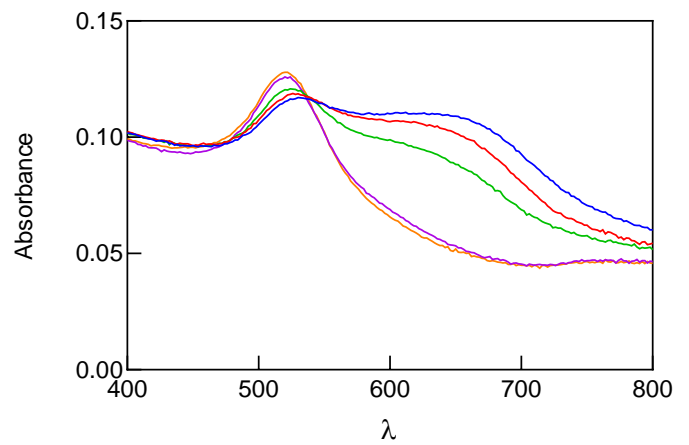

b)

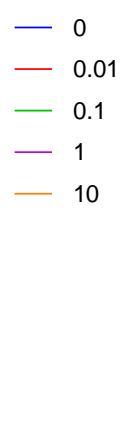

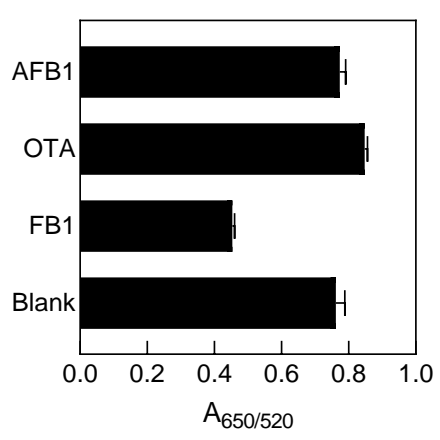

Figure 4. UV-VIS spectra of AuNP functionalized with a 96-nt aptamer previously incubated with FB1 $(0-10 \mu \mathrm{g} / \mathrm{mL})$ in $\mathrm{MgCl} 21 \mathrm{mM}$ (Binding: $30 \mathrm{~min}$ at $37^{\circ} \mathrm{C}$, Functionalization: $60 \mathrm{~min}$ at RT, Aptamer: AuNP 30:1) after the addition of $\mathrm{NaCl} 0.2 \mathrm{M}$ (a); specificity test after the incubation with buffer (blank), or FB1, aflatoxin B1 (AFB1), and ochratoxin A(OTA) at a concentration of $1.38 \mu \mathrm{M}(\mathbf{b})$.

\subsection{Analysis of AuNP functionalized with an Aptamer-FB1 complex by Asymmetric Flow Field Flow Fractionation (AF4)}

Unlike other aptasensors in which the addition of the target molecule promotes the whole DNA sequence detachment from AuNP or its dehybridization from complementary sequences, this biosensor presents the formation of an Aptamer-FB1-AuNP complex, which is more stable to particle aggregation $(\mathrm{NaCl} 0.2 \mathrm{M})$ at higher $\mathrm{FB} 1$ concentrations.

As displayed in Figure 5a, the movement of particles through the membrane occurred from small to greater size, which in the case of the aptamer-FB1-AuNP conjugate can be translated into non-aggregated to aggregated particles. Therefore, higher peaks where observed by the end of each run, where a bigger size and more aggregated particles are expected, especially at $600 \mathrm{~nm}$, where the aggregation profile is more notorious by UV-VIS. After calculating the area under the highest peak (from $20 \mathrm{~min}$ run), the limit of detection was estimated as $0.000056 \mathrm{ng} / \mathrm{mL}$ ( $56 \mathrm{fg} / \mathrm{mL}$ ). Similarly, the MALS results at $28^{\circ}$ (Figure $5 \mathrm{~b}$ ) indicated a single predominant peak towards the end of each run, with a reduction of the peak height at increasing FB1 concentrations. This signal also reported a good sensitivity with an LOD of $0.00016 \mathrm{ng} / \mathrm{mL}$. Note that these results are consistent with the notion that the conjugation is promoted by the presence of the target mycotoxin, which was visually confirmed by its red color, and now evidenced by the distribution of the different signals in AF4.

These results imply that the main role of FB1 in promoting the functionalization of AuNP involves the whole aptamer sequence, and is not only mediated through its affinity to the molecule, but also by the interaction with the rest of the DNA sequence which is not involved in binding the FB1 epitope region. 
a)

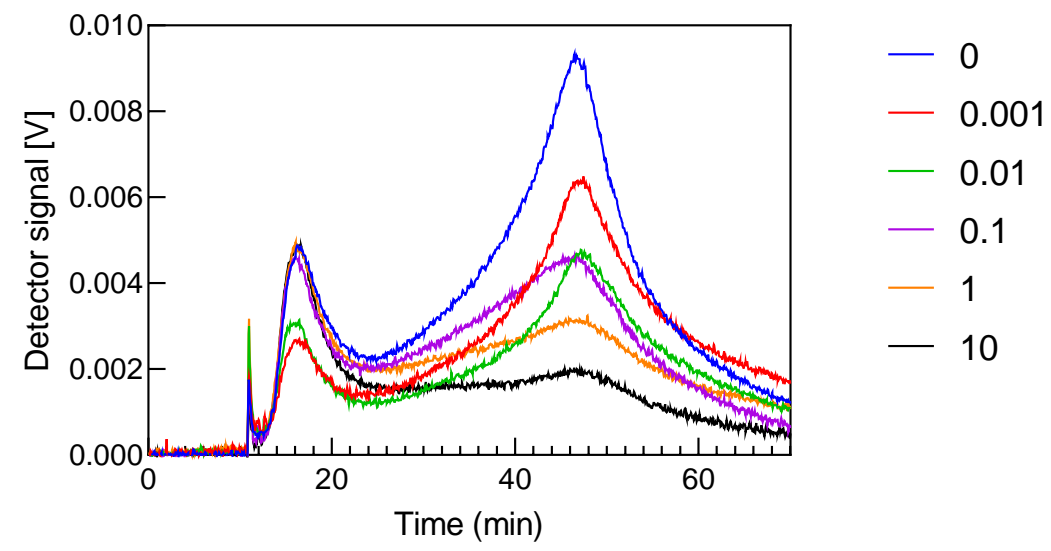

b)

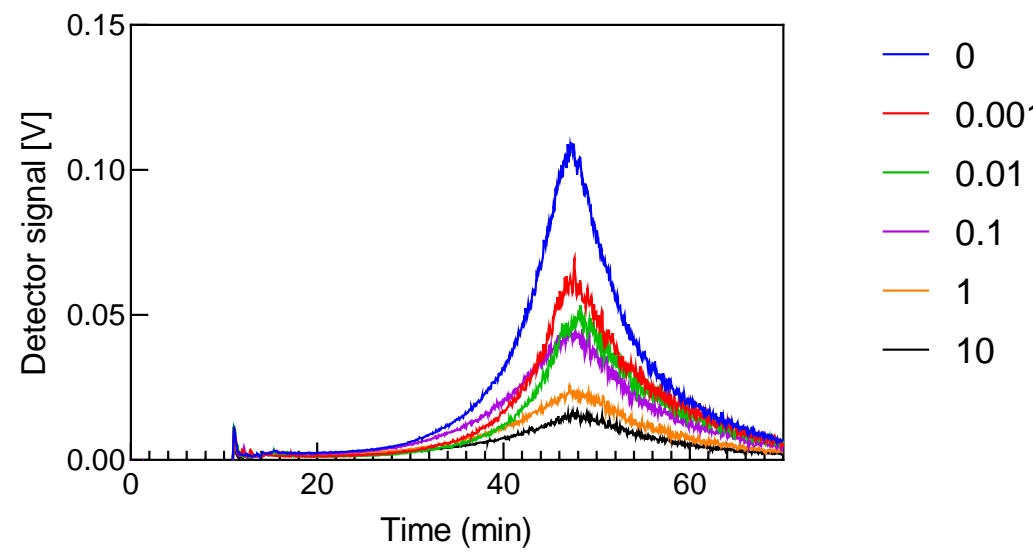

Figure 5. Aptamer-FB1-AuNP conjugate at different FB1 concentration upon addition of $\mathrm{NaCl}(0.2 \mathrm{M})$ detected by UV: $600 \mathrm{~nm}$ (a); and MALS: $28^{\circ}$ (b) signals.

\section{Conclusions}

In this work, a bulk aptasensor method was developed by incubating an aptamer with its target molecule (FB1), and afterwards with AuNP. The appropriate incubation conditions (time, temperature, buffer) derived in the formation of an aptamer-FB1-AuNP conjugate, mediated by the presence of FB1.

Even when acceptable limits of detection were found by analyzing the $\mathrm{A}_{650 / 520}$ ratio from spectrophotometric scans, the analysis of the interaction within the conjugation elements by AF4 revealed a novel potential biosensing technique with a limit of detection in the $\mathrm{fg} / \mathrm{mL}$ range, with the added novelty of utilizing a ssDNA aptamer sequence without the aid of any complementary strand.

Author Contributions: Conceptualization, V.A.M.-M.; Y.G.; Y.Y.G. and F.M.G.; methodology, V.A.M.-M. and Y.G.-E.; software, V.A.M.-M. and Y.G.-E.; validation, V.A.M.-M.; formal analysis, V.A.M.-M.; investigation, V.A.M.-M.; resources, Y.G., Y.Y.G. and F.M.G.; data curation, V.A.M.-M.; writing-original draft preparation, V.A.M.-M.; writing - review and editing, V.A.M.-M. and F.M.G.; visualization, V.A.M.-M.; supervision Y.Y.G. and F.M.G.; project administration, V.A.M.-M. and F.M.G.; funding acquisition, V.A.M.-M. and F.M.G. All authors have read and agreed to the published version of the manuscript.

Acknowledgments: Vicente A. Mirón-Mérida was awarded with a scholarship from CONACyT (Mexico) for his PhD studies.

Conflicts of Interest: The authors declare no conflict of interest. 


\section{References}

1. Rheeder, J.P.; Marasas, W.F.; Vismer, H.F. Production of fumonisin analogs by Fusarium species. Appl. Environ. Microbiol. 2002, 68, 2101-2105.

2. Ostry, V.; Malir, F.; Toman, J.; Grosse, Y. Mycotoxins as human carcinogens-The IARC Monographs classification. Mycotoxin Res. 2017, 33, 65-73.

3. Lee, S.; Kim, G.; Moon, J. Performance improvement of the one-dot lateral flow immunoassay for aflatoxin B1 by using a smartphone-based reading system. Sensors 2013, 13, 5109-5116.

4. McKeague, M.; Bradley, C.R.; Girolamo, A.D.; Visconti, A.; Miller, J.D.; DeRosa, M.C. Screening and initial binding assessment of Fumonisin B1 aptamers. Int. J. Mol. Sci. 2010, 11, 4864-4881.

5. Chen, X.; Huang, Y.; Duan, N.; Wu, S.; Xia, Y.; Ma, X.; Zhu, C.; Jiang, Y.; Ding, Z.; Wang, Z. Selection and characterization of single stranded DNA aptamers recognizing fumonisin B1. Microchim. Acta 2014, 181, 1317-1324.

6. Pandey, P.C.; Pandey, G. Synthesis of gold nanoparticles resistant to $\mathrm{pH}$ and salt for biomedical applications; functional activity of organic amine. J. Mater. Res. 2016. 31, 3313-3323.

7. Rosi, N.L.; Mirkin, C.A. Nanostructure in biodiagnostics. Chem. Rev. 2005, 105, 1547-1562.

8. Hagendorfer, H.; Kaegi, R.; Traber, J.; Mertens, S.F.L.; Scherrers, R.; Ludwig, C.; Ulrich, A. Application of an asymmetric flow field flow fractionation multi-detector approach for metallic engineered nanoparticle characterization- Prospects and limitations demonstrated on Au nanoparticles. Anal. Chim. Acta 2011, 706, 367-378.

9. Tian, H.; Sofer, Z.; Pumera, M.; Bonanni, A. Investigation on the ability of heteroatom-doped graphene for biorecognition. Nanoscale 2017, 9, 3530-3536.

10. Cheng, Z.X.; Bonanni, A. All-in-One: Electroactive Nanocarbon as Simultaneous Platform and Label for Single-Step Biosensing. Chem. Eur. J. 2018, 24, 6380-6385.

11. Chávez, J.L.; Hagen, J.A.; Kelley-Loughnane, N. Fast and selective plasmonic serotonin detection with aptamer-gold nanoparticle conjugates. Sensors 2017, 17, 681.

Publisher's Note: MDPI stays neutral with regard to jurisdictional claims in published maps and institutional affiliations.

(C) 2020 by the authors. Submitted for possible open access publication under the terms and conditions of the Creative Commons Attribution (CC BY) license (http://creativecommons.org/licenses/by/4.0/). 